\title{
Valores sociales y adicción: aplicabilidad y propiedades psicométricas del cuestionario VAL-89
}

\author{
Eduardo J. Pedrero Pérez; Gloria Rojo Mota; Álvaro Olivar Arroyo \\ CAD 4 San Blas. Instituto de Adicciones. Ayuntamiento de Madrid. \\ Enviar correspondencia a: \\ Eduardo J. Pedrero Pérez. C/Alcalá 527. 28027 MADRID. Tfno: 609587233. Fax: 917434775. C.E.: ejpedrero@yahoo.es
}

Recibido: Octubre de 2007 Aceptado: Marzo de 2008

\section{RESUMEN}

Objetivo: Estudiar las propiedades psicométricas y la aplicabilidad del cuestionario VAL-89 en población adicta que solicita tratamiento.

Método: Análisis de las propiedades psicométricas del cuestionario y su estructura factorial, mediante su aplicación a 792 sujetos de los que 365 son consumidores de sustancias que demandan tratamiento por problemas asociados al consumo y 427 eran población general.

Resultados: Se confirma la fiabilidad del cuestionario, aunque la estructura factorial no se corresponde con la original. Frente a los 10 factores originales, en nuestro estudio aparecen 12 factores: Poder, Estimulación, Sumisión, Tradición, Espiritualidad, Autosuficiencia, Hedonismo, Sociabilidad, Universalidad, Convencionalismo, Idealismo y Autorrealización. Estos factores se agrupan en función de dimensiones en forma de ejes tales como lo individual frente a lo social, la dominación sobre los otros frente a la justicia y la igualdad, las tradiciones y convenciones sociales frente a la búsqueda del placer y el disfrute $y$, por último, los grandes valores frente a la anomia.

Conclusiones: EI VAL-89 aparece como un cuestionario útil para explorar los valores sociales que más se aprecian, resultando de especial interés cuáles son los más destacables para los individuos adictos.

\section{ABSTRACT}

Objective: To study the psychometric properties of the VAL-89 questionnaire and its possible use in addict individuals who ask for treatment.

Method: Analysis of the psychometric properties of the questionnaire and its factorial structure, applying it to 792 individuals. 365 of them were substance users seeking treatment and 427 were general population.

Results: Reliability of the questionnaire is confirmed, although its factorial structure appears to be different from the original. In our study appear 12 factors, instead of the original 10. These factors are named: Power, Stimulation, Submission, Tradition, Spirituality, Self-Sufficience, Hedonism, Sociability, Universality, Convencionalism, Idealism and Self-Realization. These factors are distributed through several dimensions represented by four axis: individual-social, dominance-equality, tradition-pleasure and great values-anomie.

Conclussions: The VAL-89 questionnaire seems to be a useful tool to explore which are the more appreciated social values, being of special interest to know which are specially selected by addict individuals.

Key words: Social values, assessment, VAL-89, addiction.

Palabras clave: Valores sociales, evaluación, VAL-89, adicción.

\section{INTRODUCCIÓN}

I concepto de "valor social" hunde sus raíces E en la filosofía, pero ha adquirido en las últimas décadas un renovado interés en las Ciencias Sociales (Psicología Social, Sociología, Pedagogía) en la medida en que supone la estimación de metas y la validación de procedimientos para alcanzarlos de las diferentes culturas humanas. A pesar de su frecuente utilización en la investigación en ciencias sociales, el término valor tiene múltiples acepciones. Esta situación representa un serio problema para poder establecer un conjunto de conceptos con un significado compartido con el que poder expresar la relación entre el conocimiento sociológico o psicológico y la realidad social a la que dicho conocimiento se refiere. Al igual que otros conceptos interdisciplinares como los de representación social, actitud, creencia, etc., el concepto de valor está ubicado en un terreno conceptual 
indiferenciado con relación a conceptos afines (Garrido, 2006).

Montero (1994), tras realizar una revisión de las diferentes definiciones del concepto, señala que un valor "es un elemento o proceso mediador que relaciona las evaluaciones (componente afectivo) con la cognición (componente cognoscitivo) y produce alguna forma de comportamiento (elemento conativo)". Rokeach (1973) definió los valores como creencias personales consistentes y duraderas de carácter evaluativo. Para este autor los valores, en cuanto creencias, son elementos pertenecientes al ámbito cognitivo del sujeto y están estrechamente ligados a las funciones evaluativas de la corrección o incorrección de los más variados sucesos y acontecimientos sociales y personales. De este modo, los valores adquieren un significado especial en la medida que poseen carácter prescriptivo para la conducta de las personas: en tanto que creencias duraderas, dirigen la conducta en función de la preferencia por un modo de existencia o de conducta concreto en detrimento de otros modos de conducta y estados finales. La teoría sobre el sistema de creencias coloca los valores en el núcleo de la personalidad del individuo. Distinguió entre dos tipos: los valores finalistas, referidos o de existencia: paz, libertad, felicidad o bien común; y los valores instrumentales o relacionados con modos de conducta: valores morales. Para la estimación cuantitativa de los valores, el autor construyó el Inventario de Valores de Rokeach (Rokeach Value Survey, RVS), autoinforme de 36 items que mide 18 valores de carácter finalista y 18 de carácter instrumental.

Schwartz y Bilsky $(1987,1990)$, a partir de las propuestas de Rokeach, han elaborado un modelo teórico para el estudio de los valores personales, sosteniendo que éstos tienen una estructura universal. En este modelo se definen diez tipos básicos de valores como son: universalismo, benevolencia, tradición conformidad, seguridad, poder, logro, hedonismo, estimulación y autodirección. Este modelo ha sido contrastado en diferentes países con culturas diferentes y ha sido aplicado al estudio de diversos aspectos de la realidad social (Gouveia, Martínez, Meira y Lemos, 2001; Grad y Schwartz, 1998).

Eckersley (2005) denuncia la poca atención que la investigación ha prestado a los valores sociales dominantes como generadores de comportamientos contrarios a la salud, entre ellos el abuso de drogas, en tanto que los estudios han tendido a centrarse con mayor frecuencia en las diferencias que los grupos minoritarios mostraban. La misma carencia de estudios es observada en España (Alonso, Comas, Megías y Pedrero, 1995), aunque en los últimos años se han efectuado encuestas sociológicas que han explorado, con cuestionarios ad hoc, la relación entre los valores asumidos por los jóvenes y el consumo de sustancias (Comas, Aguinaga, Andrés, Espinosa y Ochaita, 2003;
Megías, Comas, Elzo, Megías, Navarro, Rodríguez et al., 2000; Megías, Elzo, Comas, Rodríguez, Megías, Navarro et al., 2001).

Este vacío se debe, en parte, a la carencia de instrumentos validados para la estimación de las variables objeto de estudio. El Rokeach Value Survey ha sido utilizado para estudiar los valores de consumidores de sustancias como el tabaco y el alcohol, pero desde la perspectiva de estudios de mercado (Kropp, Lavack y Holden, 1999). Muy pocos trabajos lo utilizan para el estudio de adictos y todos ellos muy alejados en el tiempo y el ámbito cultural (Mercer y Kohn, 1977; Toler, 1975). Unger, Ritt-Olson, Teran, Huang, Hoffman y Palmer (2002) construyeron un cuestionario ad hoc a partir de ítems de otras escalas, para explorar la relación entre valores y abuso de sustancias, aunque lo que consiguieron medir es, más propiamente, actitudes diferenciales en diversos grupos étnicos.

En España un estudio utilizó el Rokeach Value Survey en una muestra de adictos a heroína en tratamiento (Rojo, Valdemoro y Cervera, 1994). Pons y Berjano (1999) utilizaron el VAL-89, derivado del modelo de Rokeach modificado por Schwartz y Bilsky (1987), para estudiar una muestra de adolescentes, explorando algunas propiedades psicométricas del cuestionario, que se revelan adecuadas al objeto de estudio.

El presente trabajo tiene por objeto conocer las propiedades psicométricas y la aplicabilidad del VAL-89 en población adicta que solicita tratamiento. El conocimiento de la fiabilidad y validez factorial del cuestionario permitiría, en fases posteriores, explorar su relación con otras variables clínicas (consumo de sustancias, psicopatología, variables de personalidad, indicadores de salud) y derivar implicaciones de interés para el abordaje terapéutico y educativo de las personas con problemas relacionados con el consumo de sustancias.

\section{MATERIAL Y MÉTODO}

\section{Participantes}

Se administró el VAL-89 a una muestra, obtenida mediante muestreo consecutivo, de 365 sujetos que inician tratamiento ambulatorio por abuso/dependencia de heroína ( $N=89)$, cocaína $(N=142)$, alcohol ( $N=103$ ) o cannabis ( $N=29$ ), en un centro público del Ayuntamiento de Madrid (CAD 4 San Blas).

Adicionalmente se obtuvieron otras dos muestras:

-- 220 estudiantes universitarios que colaboraron desinteresadamente en el estudio, pertenecientes a las Universidades de Castilla La Mancha (Sociología) y Universidad Rey Juan Carlos (Terapia Ocupacional). 
-- 207 sujetos de población general, muestra obtenida mediante la técnica de bola de nieve (Goodman, 1961).

Los descriptivos de los diferentes subgrupos se presentan en la Tabla 1.

\section{Instrumentos}

Se utilizó la escala VAL-89, originalmente elaborada por Schwartz y Bilsky (1987) a partir del Rokeach Value Survey (Rokeach, 1973) y del Chinese Value Survey (Chinese Culture Connection, 1987). La versión que utilizamos es la reelaborada por Molpeceres (1991) y publicada íntegramente por Pons y Berjano (1999). El primitivo cuestionario de Rokeach solicitaba una ordenación jerárquica de cada lista de valores en base a las prioridades del sujeto, mientras que la modalidad de respuesta originaria del cuestionario de Schwartz era una escala tipo Likert con un rango de 1 a 7, en la que el sujeto debía puntuar cada uno de los valores. Hemos utilizado para el presente estudio la versión propuesta por Pons y Berjano (1999), con una modalidad de respuesta en una dimensión continua de 0 a 10 en la que el sujeto debe situarse frente a cada valor dado, lo que, como sugieren los autores, se ajusta más a la lógica del continuo aportada por Schwartz.

En el estudio citado (Pons y Berjano, 1999) el cuestionario presentó una consistencia interna, medida por el coeficiente de Cronbach, de $\nabla=0,91 \quad(0,82$ y 0,84 para cada mitad del test), y la distribución de sus resultados mostró un ajuste a la curva normal. El test se compone de 10 escalas derivadas factorialmente (en el estudio de Schwartz, 1992, pero no en la versión española), que se denominan: Autodirección, Universalidad, Benevolencia, Tradición, Conformidad, Seguridad, Poder, Logro, Hedonismo y Estimulación.
Un análisis de segundo orden de la escala orioginal (Schwartz, 1992) a partir de las puntuaciones de las escalas encontró dos dimensiones: Autobeneficio (Poder, Logro y Hedonismo) vs. Autotrascendencia (Universalidad y Benevolencia) y Apertura (Autodirección, Hedonismo y Estimulación) vs. Conservación (Tradición, Conformidad y Seguridad).

\section{Procedimiento}

Los pacientes cumplimentaron los cuestionarios una vez libres de sintomatología atribuible a la intoxicación o a la retirada de las drogas. Los estudiantes universitarios lo hicieron en el transcurso de una sesión de formación, de modo voluntario. La muestra de población general se obtuvo mediante la técnica de "bola de nieve" (Goodman, 1961).

Una submuestra de 60 sujetos, adictos en tratamiento, repitió la cumplimentación un mes después del primer pase, para estudiar la estabilidad temporal de la prueba.

Se utilizó, en primer lugar, la muestra de población general y la de estudiantes para efectuar un análisis factorial exploratorio. A continuación, se realizó un análisis factorial confirmatorio utilizando la muestra de adictos en tratamiento. Finalmente, los resultados se sumarizaron, haciendo constar en tablas los estadísticos para ítems y escalas sobre el total de la muestra.

\section{Análisis de datos}

Se utilizó el paquete estadístico SPSS 12.0 para Windows. Se efectuaron análisis factorial exploratorio de primer y segundo orden, análisis de fiabilidad de las escalas y escalamiento multidimensional ALSCAL. Se utilizó el programa AMOS 4.01 para efectuar análisis factorial confirmatorio.

Tabla 1. Descriptivos de la muestra y submuestras

\begin{tabular}{|l|c|c|c|c|c|c|c|c|c|c|}
\cline { 2 - 12 } \multicolumn{1}{c|}{} & \multicolumn{4}{c|}{ Edad } & \multicolumn{9}{c|}{ Nivel de estudios } & \multicolumn{2}{c|}{ Sexo } \\
\cline { 2 - 13 } & Media & D.T. & \multicolumn{2}{c|}{ Rango } & $\begin{array}{c}\text { SIN ESTUDIOS } \\
\text { PRIMARIOS }\end{array}$ & $\begin{array}{c}\text { EGB } \\
\text { PRIMARIA }\end{array}$ & $\begin{array}{c}\text { SECUNDARIA } \\
\text { BUP COU }\end{array}$ & UNIVERSITARIOS & VARONES & MUJERES \\
\hline ADICTOS & 35.1 & 9.2 & 16 & 61 & $11.8 \%$ & $46.3 \%$ & $31.0 \%$ & $11.0 \%$ & $76.2 \%$ & $23.8 \%$ \\
\hline Heroína & 37.6 & 6.4 & 17 & 53 & $18.0 \%$ & $34.8 \%$ & $40.4 \%$ & $6.7 \%$ & $74.2 \%$ & $25.8 \%$ \\
\hline Cocaína & 31.3 & 8.2 & 18 & 61 & $9.8 \%$ & $52.4 \%$ & $28.0 \%$ & $9.8 \%$ & $79.7 \%$ & $20.3 \%$ \\
\hline Alcohol & 40.8 & 7.9 & 22 & 60 & $12.5 \%$ & $44.2 \%$ & $27.9 \%$ & $15.4 \%$ & $68.3 \%$ & $31.7 \%$ \\
\hline Cannabis & 25.4 & 9.4 & 16 & 47 & - & $58.6 \%$ & $27.6 \%$ & $13.8 \%$ & $93.1 \%$ & $6.9 \%$ \\
\hline ESTUDIANTES & 20.7 & 2.8 & 18 & 43 & - & - & - & $100 \%$ & $13.6 \%$ & $8.4 \%$ \\
\hline $\begin{array}{l}\text { POBLACIÓN } \\
\text { GENERAL }\end{array}$ & 37.8 & 10.6 & 16 & 70 & $2.4 \%$ & $9.2 \%$ & $24.2 \%$ & $64.3 \%$ & $35.3 \%$ & $64.7 \%$ \\
\hline
\end{tabular}




\section{RESULTADOS}

La fiabilidad del cuestionario completo, medida por el coeficiente de Cronbach, es $\nabla=0,91$ ( $\nabla=0,91$ para las submuestras de población general y adictos y $\nabla=0,90$ para la de estudiantes universitarios); el coeficiente $\mathbb{\square}$ estandarizado de los items es de 0,92 y el coeficiente test retest al mes es de $r=0,84$. Los datos son prácticamente idénticos a los observados por Pons y Berjano (1999).

Se procedió a efectuar un análisis factorial exploratorio sobre los 56 ítems del cuestionario. La medida de adecuación muestral Kaiser-Meyer-Olkin fue de 0,90 y el test de esfericidad de Bartlett mostró una significación de $p<0,001$, lo que aconsejó seguir adelante con este procedimiento. Se llevó a cabo un análisis de componentes principales. Dado que no se esperaba ortogonalidad entre los diversos valores explorados, sino una fuerte correlación entre ellos, se optó por una rotación oblicua (Oblimin). Se obtuvo una solución de 12 factores con autovalores mayores de 1, que explicaban en total un $54,7 \%$ de la varianza del test (Tabla 2). La distribución de los ítems en estos componentes apenas presentó similitud con la presentada en el estudio previo con adolescentes (Pons y Berjano, 1999). Por ello, se procedió a renombrar las escalas en función de los ítems observados en el presente estudio. En la Tabla 3 se muestran los estadísticos de las nuevas escalas y la denominación por la que se ha optado. La consistencia interna de cada escala es variable, estando en torno a $\triangle=0,75$ para las escalas con 6 o 7 ítems, y en torno a $\otimes=0,55$ para las que sólo cuentan con 3 o 4 ítems. La consistencia encontrada para cada escala es similar en las dos submuestras (adictos y no adictos). En la Tabla 4 se muestran los ítems que componen cada escala, haciendo constar las cargas factoriales en la escala de referencia.

Tabla 2. Componentes encontrados mediante análisis de componentes principales y rotación Oblimin de los ítems del VAL-89, y varianza total explicada.

\begin{tabular}{ccccc}
\hline Componente & Total & \% de la Varianza & \% Acumulado & Rotación \\
\hline 1 & 10,60 & 18,94 & 18,94 & 5,58 \\
2 & 4,10 & 7,32 & 26,26 & 3,67 \\
3 & 3,10 & 5,54 & 31,80 & 3,14 \\
4 & 2,29 & 4,10 & 35,90 & 4,63 \\
5 & 1,74 & 3,10 & 39,00 & 1,72 \\
6 & 1,50 & 2,68 & 41,68 & 3,25 \\
7 & 1,45 & 2,59 & 44,26 & 2,53 \\
8 & 1,36 & 2,42 & 46,68 & 2,44 \\
9 & 1,24 & 2,21 & 48,89 & 3,45 \\
11 & 1,11 & 1,98 & 50,88 & 3,24 \\
12 & 1,07 & 1,91 & 52,79 & 2,78 \\
\hline
\end{tabular}

Tabla 3. Estadísticos de las escalas del VAL-89, obtenidos mediante análisis factorial exploratorio y confirmatorio.

\begin{tabular}{lccccccccc} 
& & & \multicolumn{3}{c}{$\nabla$ de Cronbach } & \multicolumn{3}{c}{ Varianza } \\
\cline { 3 - 9 } & $\mathrm{n}^{0}$ de ítems & \% varianza & Total & No adictos & Adictos & Estimada & D.E. & R.C. & Sig. \\
\hline Autorrealización & 7 & 18,9 & 0,75 & 0,72 & 0,78 & 0,52 & 0,08 & 6,69 & 0,000 \\
Poder & 6 & 7,3 & 0,77 & 0,77 & 0,77 & 2,30 & 0,30 & 7,60 & 0,000 \\
Estimulación & 4 & 5,5 & 0,76 & 0,79 & 0,73 & 3,88 & 0,34 & 11,46 & 0,000 \\
Universalidad & 4 & 4,1 & 0,76 & 0,73 & 0,78 & 3,28 & 0,29 & 11,45 & 0,000 \\
Convencionalismo & 7 & 3,1 & 0,73 & 0,77 & 0,70 & 1,33 & 0,18 & 7,30 & 0,000 \\
Sumisión & 4 & 2,7 & 0,76 & 0,52 & 0,55 & 0,94 & 0,18 & 5,29 & 0,000 \\
Tradición & 3 & 2,6 & 0,55 & 0,56 & 0,53 & 1,13 & 0,14 & 8,01 & 0,000 \\
Espiritualidad & 4 & 2,4 & 0,52 & 0,52 & 0,52 & 1,78 & 0,26 & 6,99 & 0,000 \\
Autosuficiencia & 3 & 2,2 & 0,51 & 0,49 & 0,51 & 0,58 & 0,15 & 4,00 & 0,000 \\
Idealismo & 4 & 2,0 & 0,55 & 0,55 & 0,54 & 0,79 & 0,12 & 6,83 & 0,000 \\
Hedonismo & 3 & 1,9 & 0,56 & 0,59 & 0,53 & 1,35 & 0,18 & 7,48 & 0,000 \\
Sociabilidad & 7 & 1,9 & 0,72 & 0,72 & 0,70 & 0,70 & 0,12 & 6,08 & 0,000 \\
\hline
\end{tabular}

NOTA: C.F. = carga factorial; P.R. = pesos de regresión; D.E. = Desviación estándar; R.C. =Ratio crítica. 
Tabla 4. Factores encontrados en el VAL-89 mediante análisis factorial exploratorio (rotación: Oblimin) y patrón de covariación obtenido mediante análisis factorial confirmatorio.

\begin{tabular}{|c|c|c|c|c|c|c|}
\hline FACTOR ÍTEM & FACTORES & C.F. & P.R. & D.E. & R.C. & $p$ \\
\hline 1 & AUTORREALIZACIÓN & 1 & & & & \\
\hline 2 & Armonía interior (en paz conmigo mismo/a) & 0,51 & 1,00 & & & \\
\hline 10 & Sentido en la vida (tener un objetivo en la vida) & 0,71 & 1,45 & 0,13 & 11,39 & 0,000 \\
\hline 14 & Respeto a mi mismo (creencia en mi valor personal) & 0,70 & 1,51 & 0,13 & 11,33 & 0,000 \\
\hline 20 & Autodisciplina (autocontrol, resistencia a la tentación) & 0,55 & 1,65 & 0,16 & 10,35 & 0,000 \\
\hline 34 & Ambicioso (trabajador esforzado, con aspiraciones) & 0,58 & 1,69 & 0,16 & 10,41 & 0,000 \\
\hline 41 & Escogiendo mis metas (seleccionar mis propósitos) & 0,62 & 1,74 & 0,15 & 11,68 & 0,000 \\
\hline 45 & Honesto (sincero, auténtico) & 0,44 & 1,29 & 0,11 & 11,32 & 0,000 \\
\hline 2 & PODER & 2 & & & & \\
\hline 3 & Poder social (control sobre los otros, dominio) & 0,70 & 1,00 & & & \\
\hline 12 & Fortuna (posesiones materiales, dinero) & 0,58 & 0,94 & 0,08 & 12,18 & 0,000 \\
\hline 27 & Autoridad (el derecho de liderar o mandar) & 0,76 & 1,12 & 0,09 & 12,71 & 0,000 \\
\hline 39 & Influyente (con impacto en las personas y acontecimientos) & 0,58 & 0,91 & 0,08 & 11,99 & 0,000 \\
\hline 46 & Cuidadoso de mi imagen pública (proteger mi reputación) & 0,45 & 0,88 & 0,08 & 11,57 & 0,000 \\
\hline 55 & Triunfador, con éxito (conseguir los objetivos sociales) & 0,55 & 1,05 & 0,08 & 12,80 & 0,000 \\
\hline 3 & ESTIMULACIÓN & 3 & & & & \\
\hline 9 & Una vida excitante (experiencias estimulantes) & 0,69 & 1,00 & & & \\
\hline 16 & Creatividad (ser único, con imaginación) & 0,50 & 0,65 & 0,05 & 13,44 & 0,000 \\
\hline 25 & Una vida variada (llena de desafíos, novedades y cambios) & 0,75 & 0,89 & 0,05 & 17,28 & 0,000 \\
\hline 37 & Audaz (que procura la aventura y el riesgo) & 0,78 & 0,93 & 0,05 & 17,61 & 0,000 \\
\hline 4 & UNIVERSALIDAD & 4 & & & & \\
\hline 24 & Unión con la naturaleza (integración con la naturaleza) & 0,81 & 1,00 & & & \\
\hline 26 & Sabiduría (una comprensión madura de la vida) & 0,51 & 0,49 & 0,04 & 12,79 & 0,000 \\
\hline 29 & Un mundo de belleza (belleza de la naturaleza y de las artes) & 0,74 & 1,04 & 0,05 & 19,50 & 0,000 \\
\hline 38 & Protector del medio ambiente (conservador de la naturaleza) & 0,81 & 0,82 & 0,05 & 15,75 & 0,000 \\
\hline 5 & CONVENCIONALISMO & 5 & & & & \\
\hline 7 & Sentido de pertenencia (sentimiento de que le importo a los demás) & $-0,73$ & 1,00 & & & \\
\hline 8 & Orden social (estabilidad social) & $-0,55$ & 1,12 & 0,09 & 12,09 & 0,000 \\
\hline 22 & Seguridad familiar (seguridad para las personas que amo) & $-0,56$ & 0,59 & 0,05 & 11,00 & 0,000 \\
\hline 23 & Reconocimiento social (Respeto, aprobación de los demás) & $-0,61$ & 1,07 & 0,09 & 11,74 & 0,000 \\
\hline 28 & Amistad verdadera (amigos próximos que me apoyan) & $-0,59$ & 0,66 & 0,07 & 9,34 & 0,000 \\
\hline 33 & Leal (fiel a mis amigos y a mi grupo de pertenencia) & $-0,50$ & 0,78 & 0,07 & 11,34 & 0,000 \\
\hline 56 & Limpio (aseado y arreglado) & $-0,43$ & 0,69 & 0,06 & 11,31 & 0,000 \\
\hline
\end{tabular}

NOTA: C.F. = carga factorial; P.R. = pesos de regresión; D.E. = Desviación estándar; R.C. = Ratio crítica. 
Tabla 4. (continuación)

\begin{tabular}{|c|c|c|c|c|c|c|}
\hline 6 & ESPIRITUALIDAD & 6 & & & & \\
\hline 6 & Una vida espiritual (énfasis en las cosas espirituales y no materiales) & 0,80 & 1,00 & & & \\
\hline 19 & Amor maduro (profunda intimidad emocional y espiritual) & 0,67 & 0,62 & 0,09 & 6,99 & 0,000 \\
\hline 21 & Distanciamiento (de las cosas mundanas) & 0,52 & 1,19 & 0,13 & 9,39 & 0,000 \\
\hline 51 & Devoto (dedicado a la fé y a las creencias religiosas) & 0,55 & 0,88 & 0,11 & 7,83 & 0,000 \\
\hline 7 & SUMISIÓN & 7 & & & & \\
\hline 32 & Moderado (evitar los extremos en los sentimientos y acciones) & $-0,54$ & 1,00 & & & \\
\hline 36 & Humilde (modesto, que pasa desapercibido) & $-0,55$ & 1,15 & 0,14 & 8,52 & 0,000 \\
\hline 44 & Aceptar mi vida (sumiso a las circunstancias de la vida) & $-0,63$ & 0,93 & 0,14 & 6,75 & 0,000 \\
\hline 47 & Obediente (cumplidor de mis deberes y obligaciones) & $-0,52$ & 1,67 & 0,17 & 10,14 & 0,000 \\
\hline 8 & TRADICIÓN & 8 & & & & \\
\hline 11 & Cortesía (educación, buenas maneras) & 0,48 & 1,00 & & & \\
\hline 13 & Seguridad nacional (protección de mi nación frente a los enemigos) & 0,62 & 1,50 & 0,13 & 11,24 & 0,000 \\
\hline 18 & $\begin{array}{r}\text { Respeto por la tradición (preservación de costumbres establecidas } \\
\text { hace mucho tiempo) }\end{array}$ & 0,63 & 1,34 & 0,13 & 10,62 & 0,000 \\
\hline 9 & AUTOSUFICIENCIA & 9 & & & & \\
\hline 15 & Reciprocidad de favores (evitar ser deudor de alguien) & $-0,78$ & 1,00 & & & \\
\hline 42 & Saludable (no tener dolencias físicas o mentales) & $-0,52$ & 1,27 & 0,17 & 7,33 & 0,000 \\
\hline 43 & Capaz (competente, eficaz, eficiente) & $-0,52$ & 1,76 & 0,23 & 7,69 & 0,000 \\
\hline 10 & IDEALISMO & 10 & & & & \\
\hline 1 & Igualdad (oportunidades iguales para todos) & 0,62 & 1,10 & 0,10 & 11,42 & 0,000 \\
\hline 17 & Un mundo en paz (libre de guerras y conflictos) & 0,56 & 1,26 & 0,11 & 11,42 & 0,000 \\
\hline 30 & Justicia social (ausencia de la injusticia, ayuda al más débill) & 0,75 & 0,58 & 0,10 & 5,66 & 0,000 \\
\hline 31 & Independiente (autosuficiente, autoconfiado) & 0,33 & & & & \\
\hline 11 & HEDONISMO & 11 & & & & \\
\hline 4 & Placer (satisfacción de deseos) & 0,70 & 1,00 & & & \\
\hline 5 & Libertad (de acción y pensamiento) & 0,60 & 0,50 & 0,05 & 9,20 & 0,000 \\
\hline 50 & Gozar de la vida (gusto por comer, sexualidad, diversión...) & 0,58 & 1,03 & 0,09 & 11,07 & 0,000 \\
\hline 12 & SOCIABILIDAD & 12 & & & & \\
\hline 35 & Abierto (tolerante con diferentes ideas y creencias) & $-0,41$ & 1,00 & & & \\
\hline 40 & Respetuoso (que muestra respeto, honroso) & $-0,45$ & 1,12 & 0,10 & 10,78 & 0,000 \\
\hline 48 & Inteligente (lógico, racional) & $-0,61$ & 1,18 & 0,12 & 9,94 & 0,000 \\
\hline 49 & Servicial (que trabaja para el bienestar de los demás) & $-0,48$ & 1,42 & 0,14 & 10,39 & 0,000 \\
\hline 52 & Responsable (digno de confianza) & $-0,58$ & 1,48 & 0,13 & 11,35 & 0,000 \\
\hline 53 & Curioso (interesado en todo, explorador) & $-0,55$ & 0,80 & 0,12 & 6,80 & 0,000 \\
\hline 54 & Que perdona (disculpa a los demás) & $-0,54$ & 1,15 & 0,12 & 9,76 & 0,000 \\
\hline
\end{tabular}

NOTA: C.F. = carga factorial; P.R. = pesos de regresión; D.E. = Desviación estándar; $R$. C. = Ratio crítica 
A continuación, se procedió a realizar un análisis factorial de $2^{\circ}$ orden a partir de las puntuaciones factoriales obtenidas. Puesto que no asumimos ortogonalidad entre las dimensiones del cuestionario, optamos por el método de regresión y efectuamos una rotación Oblimin. Los resultados se muestran en la Tabla 5. Se obtuvieron 4 dimensiones. La primera de ellas sitúa en polos opuestos a los valores de Autorrealización y los de Sociabilidad, representando, aparentemente, una dimensión individualista/social. La segunda dimensión sitúa en polos opuestos los valores de Poder y los Idealistas, estando estos últimos referidos a cuestiones como la justicia, la paz, la igualdad y la independencia, y aquellos otros, los de Poder, centrados en el dominio de los otros, el triunfo personal y la influencia sobre los demás. La tercera dimensión se polariza entre valores que priorizan las tradiciones y aquellos otros que se decantan por el placer y el disfrute de la vida. Finalmente, la cuarta dimensión agrupa, sin polaridad opuesta, los grandes valores: Espiritualidad, Estimulación (aventura, novedad, curiosidad), Universalidad e Idealismo.

Tabla 5. Análisis factorial exploratorio de $2^{\circ}$ orden sobre los componentes del VAL-89 (método de regresión; análisis de componentes principales con rotación Oblimin).

Componentes

\begin{tabular}{lcccc}
\hline VAL-89 & 1 & 2 & 3 & 4 \\
\hline Autorrealización & $\mathbf{0 , 6 6}$ & 0,06 & $-0,07$ & 0,06 \\
Convencionalismo & $-0,63$ & 0,10 & 0,00 & $-0,04$ \\
Sociabilidad & $-\mathbf{0 , 5 8}$ & 0,02 & $-0,09$ & $-0,16$ \\
Autosuficiencia & $-\mathbf{0 , 5 4}$ & $-0,31$ & $-0,10$ & 0,05 \\
Universalidad & $\mathbf{0 , 4 9}$ & $-0,18$ & 0,43 \\
Sumisión & $-0,47$ & 0,05 & $-0,07$ \\
Poder & 0,14 & 0,36 & 0,10 \\
Idealismo & 0,36 & 0,04 & 0,36 \\
Tradición & 0,22 & 0,16 & $\mathbf{0}$ & -02 \\
Hedonismo & 0,35 & 0,56 & $-0,01$ \\
Espiritualidad & $-0,06$ & 0,16 & $\mathbf{0 , 6 0}$ & $\mathbf{0 , 7 6}$ \\
Estimulación & 0,07 & $-0,07$ & $\mathbf{0 , 5 0}$ \\
\hline
\end{tabular}

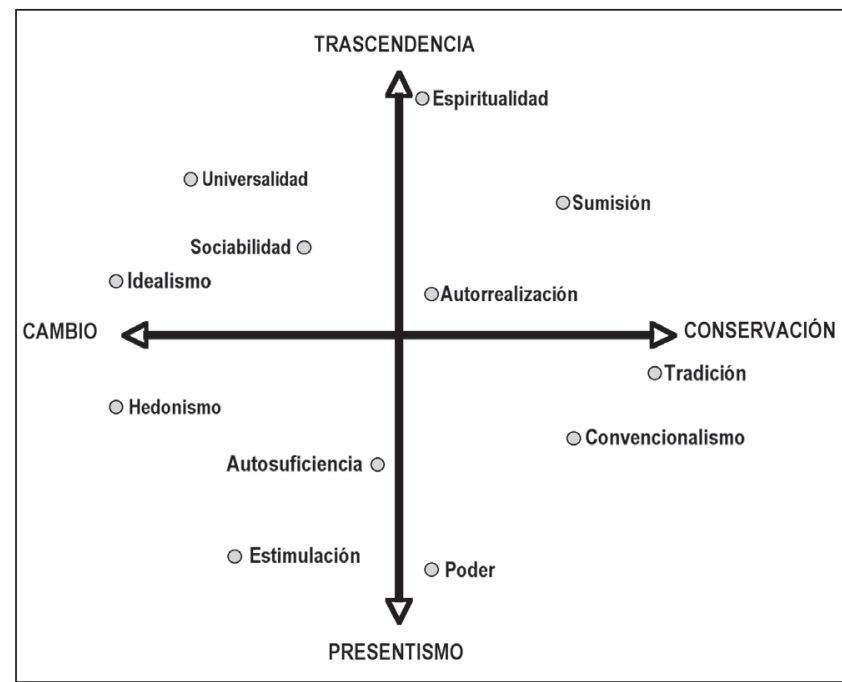

Figura 1. Escalamiento bidimensional de las escalas de VAL-89.
Para conocer las relaciones de proximidad y distancia que mantienen entre sí estas dimensiones, se efectuó un escalamiento en dos dimensiones (método PROXSCAL) a partir de la distancia euclídea de las puntuaciones (convergencia de S-stress $\mathrm{p}<0,0001$; $p<0,0001$ ). El modelo gráfico resultante (Figura 1) es perfectamente coherente con el propuesto por los autores y validado en estudios posteriores (Menezes y Campos, 1997). Las escalas se distribuyen en torno a 2 ejes principales: Trascendencia vs. Presentismo y Conservación vs. Cambio.

Por último se procedió a efectuar un análisis factorial confirmatorio de la estructura encontrada sobre la muestra de adictos en tratamiento. Utilizamos el método de estimación de máxima probabilidad (Hu \& Bentler, 1995). Para evaluar el ajuste del modelo segui- 
mos recomendaciones de los autores, que sugieren la conveniencia de emplear múltiples indicadores. Específicamente, utilizamos el estadístico $\bigotimes^{2}$, la razón de $\bigotimes^{2}$ sobre los grados de libertad, el índice de ajuste comparativo y el índice de ajuste relativo. Comparamos el modelo obtenido mediante los procedimientos recién expuestos con el modelo teórico formulado por Pons y Berjano (1999). El procedimiento confirma la bondad de ajuste del modelo $\left(\nabla^{2}=3545,01 ;\right.$ g.I.=1418; CMIN/ $D F=2.5 ; R F I=0,93 ; C F I=0,96 ; \operatorname{RMSEA}=0,32)$, no admitiendo como viable la estructura propuesta por Berjano y Pons al fallar en la definición de la matriz de covarianza implícita entre todas las dimensiones teóricas de su modelo. Finalmente, en la Tabla 6 se hacen constar los coeficientes de correlación y de regresión entre las escalas derivadas factorialmente.

Tabla 6. Coeficientes de correlación p de Pearson (parte inferior) y de regresión $\mathbf{r}^{2}$ (parte superior) entre las escalas del VAL-89.

\begin{tabular}{|c|c|c|c|c|c|c|c|c|c|c|c|c|}
\hline & $\frac{\bar{\phi}}{\overline{0}}$ & 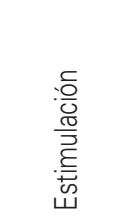 & 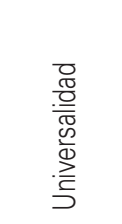 & 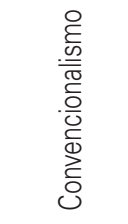 & 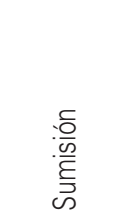 & 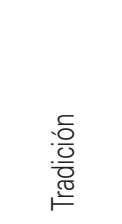 & 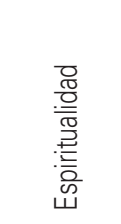 & 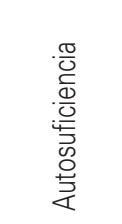 & 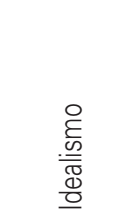 & 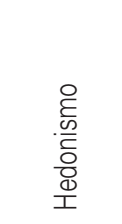 & 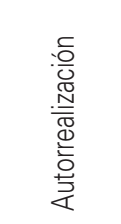 & $\begin{array}{l}\text { ㅁ } \\
\frac{\pi}{\overline{0}} \\
\frac{\overline{0}}{\overline{0}} \\
\frac{\pi}{8} \\
0\end{array}$ \\
\hline Poder & 1 & $0,18 * * *$ & 0,01 & $0,13^{* *}$ & 0,04 & $0,10^{* *}$ & 0,03 & $0,08^{*}$ & 0,00 & $0,08^{*}$ & $0,14^{* * *}$ & 0,05 \\
\hline Estimulación & $0,42 * * *$ & 1 & 0,06 & 0,04 & 0,00 & 0,00 & 0,02 & 0,04 & 0,04 & $0,18 * * *$ & 0,05 & $0,07^{*}$ \\
\hline Universalidad & $0,11 * *$ & $0,25 * * *$ & 1 & $0,09 *$ & $0,08^{*}$ & $0,10^{* *}$ & $0,10 * *$ & 0,05 & $0,18 * * *$ & 0,04 & $0,10^{* *}$ & $0,22 * * *$ \\
\hline Convencionalismo & $0,36 * * *$ & $0,19 * * *$ & $0,30 * * *$ & 1 & $0,20 * * *$ & $0,14 * * *$ & $0,07^{*}$ & $0,19 * * *$ & $0,16^{* * *}$ & $0,10^{* *}$ & $0,30 * * *$ & $0,31 * * *$ \\
\hline Sumisión & $0,19 * * *$ & 0,04 & $0,29 * *$ & $0,45 * * *$ & 1 & $0,16 * * *$ & $0,12 * *$ & $0,12^{* *}$ & $0,09 *$ & 0,02 & $0,24 * * *$ & $0,24 * * *$ \\
\hline Tradición & $0,31 * * *$ & 0,06 & $0,31 * * *$ & $0,38 * * *$ & $0,40 * * *$ & 1 & $0,09^{*}$ & 0,05 & 0,03 & 0,00 & $0,13 * *$ & $0,08^{*}$ \\
\hline Espiritualidad & $0,16 * *$ & $0,15 * * *$ & $0,31 * * *$ & $0,27 * *$ & $0,34 * * *$ & $0,30 * * *$ & 1 & 0,04 & 0,05 & 0,00 & $0,13 * *$ & $0,12 * *$ \\
\hline Autosuficiencia & $0,28 * * *$ & $0,20^{* * *}$ & $0,23 * * *$ & $0,44^{* * *}$ & $0,35 * * *$ & $0,22 * * *$ & $0,19 * * *$ & 1 & $0,12^{* *}$ & $0,07^{*}$ & $0,27 * * *$ & $0,18^{* * *}$ \\
\hline Idealismo & $0,07^{*}$ & $0,21 * * *$ & $0,42 * * *$ & $0,40 * * *$ & $0,30 * * *$ & $0,17^{* * *}$ & $0,22 * * *$ & $0,35 * * *$ & 1 & $0,09 *$ & $0,19 * * *$ & $0,25^{* * *}$ \\
\hline Hedonismo & $0,29 * * *$ & $0,43^{* * *}$ & $0,20 * *$ & $0,32 * * *$ & $0,14^{* * *}$ & 0,07 & 0,06 & $0,26 * *$ & $0,30 * * *$ & 1 & $0,07^{*}$ & $0,10^{*}$ \\
\hline Autorrealización & $0,38^{* * *}$ & $0,23^{* * *}$ & $0,32 * * *$ & $0,55^{* * *}$ & $0,49 * * *$ & $0,36^{* * *}$ & $0,36^{* * *}$ & $0,52 * * *$ & $0,44^{* * *}$ & $0,27^{* * *}$ & 1 & 0,31 \\
\hline Sociabilidad & $0,22 * * *$ & $0,26 * * *$ & $0,47 * * *$ & $0,56 * * *$ & $0,49 * * *$ & $0,28 * * *$ & $0,35 * * *$ & $0,43 * * *$ & $0,50 * * *$ & $0,32 * * *$ & $0,56 * *$ & 1 \\
\hline
\end{tabular}

NOTA: *** $p<0,001 ;{ }^{*} p<0,01 ;{ }^{*} p<0,05$.

\section{DISCUSIÓN}

Los resultados nos informan de que el VAL-89 es una prueba fiable, replicando, en su totalidad, los datos obtenidos por Pons y Berjano (1999). Puesto que en aquel trabajo se estudió una muestra de adolescentes y en el presente una de estudiantes universitarios, otra de población general y otra de población clínica, y que, como hemos visto, la consistencia interna del cuestionario es similar en todas ellas, podemos considerarlo una escala fiable para uso en diversas poblaciones. La medida, además, parece resultar estable en el tiempo.
Los datos del presente estudio no coinciden, sin embargo, con la estructura de escalas propuesta por Schwartz a partir del análisis factorial. Si él encontraba 10 dimensiones, nuestros datos apuntan a una solución de 12 factores en los que la distribución de los ítems difiere sensiblemente de la apreciada en aquel estudio. Estos 12 factores componen 12 escalas cuya fiabilidad oscila en función de los ítems que la compongan, mostrando valores adecuados cuando están compuestas por 607 ítems, pero más precarios cuando las escalas originadas cuentan con sólo 3 o 4. Versiones posteriores del cuestionario deberían contemplar el reforzamiento de estas escalas con nuevos ítems que incrementaran su fiabilidad. 
El análisis factorial de $2^{\circ}$ orden nos muestra una estructura dimensional compleja. La mayor parte de las escalas muestran pesos factoriales considerables en varias de las dimensiones encontradas, evidenciando que nos encontramos en un territorio intrincado, el de los valores asumidos por las personas, en el que es difícil trazar mapas que separen unas valoraciones de otras. Sí parece encontrarse que tales valoraciones oscilan entre lo individual y lo social, entre la dominación sobre los otros y el idealismo de la justicia y la igualdad, entre la seguridad sustentada en las tradiciones y convenciones sociales y la búsqueda del placer y el disfrute, y entre los grandes valores y la anomia.

El escalamiento bidimensional nos aclara sustancialmente el panorama. Los dos ejes principales propuestos por los autores se replican en nuestro estudio: los valores de Tradición suponen el polo de Conservación y en su entorno se sitúan los de Sumisión, más próximos a valores espirituales (posiblemente de tipo religioso), y el Convencionalismo, más proclive al respeto de las convenciones sociales presentes. En el polo opuesto, el de Cambio, se sitúan los que promueven un cambio más trascendente, Idealismo, y los que indican un cambio más pragmático, Hedonismo. En el otro eje, los valores de Espiritualidad se sitúan en el extremo de Trascendencia y próximos a los de Universalidad (cercanos al Idealismo) y los de Sumisión (más cercanos al conservacionismo). En el extremo opuesto, los valores de Poder y Estimulación se muestran más próximos al Presentismo y desprovistos de elementos trascendentes.

Los valores de autorrealización ocupan un lugar muy próximo al centro de ambos ejes, representando la equidistancia entre todos los extremos, mientras que los de Autosuficiencia parecen representar una modalidad de los anteriores más individualista y centrada en metas pragmáticas. La Sociabilidad se sitúa en el punto medio del cuadrante de cambio y Trascendencia y muy próxima a valores de Universalidad e Idealismo, y se opone a la mera aceptación de usos y costumbres, apuntando a metas más ambiciosas para el individuo y el grupo.

Como puede observarse en la Tabla 8, todas las escalas presentan correlaciones entre sí, de mayor o menor magnitud, pero positivas en todos los casos. Este efecto deriva de la propia estructura del cuestionario, en el que todos los valores son puntuados entre 0 y 10 puntos, sin que una puntuación en uno suponga el descenso en la puntuación de cualquier otro. Es probable que cuando una persona puntúe 10 en Poder puntúe 2 en Sumisión, lo que supone una correlación de $r=0,2$. En definitiva, lo que finalmente nos ofrece este cuestionario, tal y como ha sido estructurado, no es un plano de relación entre valores, sino lo que en el ideario popular se entiende como una escala de valores. Es decir, una ordenación de los valores propuestos en función de la preferencia personal.

La estructura del cuestionario, explorada en la población general, resulta confirmada posteriormente en la muestra clínica. La estructura encontrada mejora la propuesta por Pons y Berjano (1999), a partir de la obtenida factorialmente por Schwartz (1992) con diferente sistema de puntuación. Sin embargo, observando los pesos de regresión de los ítems (Tabla 4) observamos que la exclusión de algunos ítems, que presentan valores muy bajos (p.e., ítem 26, P.R.=0,49; ítem 5, P.R.=0,50) mejoraría el ajuste del modelo, si bien, al pertenecer a escalas integradas por muy pocos ítems, sería acusado por la fiabilidad de éstas. Posteriores versiones del cuestionario deberían ensayar nuevos ítems que exploraran el campo teórico de cada valor e incrementaran la fiabilidad de las escalas más precarias y, en consecuencia, la estructura general de la prueba.

No obstante, es preciso hacer constar la dificultad que implica la medición de los valores sociales. Al no existir evidencia empírica para un modelo teórico, tampoco contamos con un inventario de los posibles dominios de la valoración social, ni tampoco para el el escaneo exhaustivo de las muestras que compondrían tales dominios. El modelo de Rokeach está elegantemente propuesto desde lo teórico, pero no ha acumulado suficiente apoyo empírico como para definir una jerarquía de dominios de evaluación social, ni una taxonomía de valores. Una dificultad añadida es que, por definición, los valores son privativos de cada cultura, lo que supone que por más que definamos dominios de orden superior, o valores universales (Schwartz, 1987, 1992) los valores de inferior rango se imbrican entre sí y con aquellos de maneras diversas, lo que, en último término, es, sin más, lo que llamamos cultura.

A pesar de ello, la consideración de los valores como determinantes distales de la conducta hace necesario profundizar en su estudio, tanto más cuanto que algunas problematicas son indefinibles desvinculadas de sus determinantes psicosociales. Por ello, contar con instrumentos sólidos, fiables y estructuralmente válidos es una necesidad de primer orden. El VAL-89 muestra adecuadas propiedades para la exploración de los valores sociales asumidos por sujetos de población general y de población clínica, lo que posibilita y aconseja su utilización. Futuros estudios deberán afinar en la definición de las escalas, la incorporación de otras actualmente ausentes, la distribución equitativa de ítems y la validez externa de las medidas. 


\section{REFERENCIAS}

Alonso, C., Comas, D., Megías, E. y Pedrero, Y. (1995). Los valores de los adolescentes y el consumo de drogas. Adicciones, 7, 77-90.

Chinese Culture Connection (1987). Chinese values and the search for culture-free dimensions of culture. Journal of Cross-Cultural Psychology, 18, 143-164. Citado en J. Pons y E. Berjano, El consumo abusivo de alcohol en la adolescencia: un modelo explicativo desde la psicología social. Madrid: Plan Nacional Sobre Drogas, 1999.

Comas, D., Aguinaga, J., Andrés, F., Espinosa, A. y Ochaita, E. (2003). Jóvenes y estilos de vida: valores y riesgos en los jóvenes urbanos. Madrid: FAD-INJUVE.

Eckersley, R.M. (2005). 'Cultural fraud': the role of culture in drug abuse. Drug and Alcohol Review, 24, 157-163.

Garrido, A. (2006). Valores. En Diccionario Crítico de Ciencias Sociales, Universidad Complutense de Madrid. Disponible en URL [accedido 18-03-2006]: http://www. ucm.es/info/eurotheo/diccionario/N/valores.htm.

Goodman, L. (1961). Snowball sampling. Annals of Mathematical Statistics, 32, 148.

Gouveia, V.V., Martínez, E., Meira, M. y Lemos, T. (2001). A estrutura e o conteúdo universais dos valores humanos: análise fatorial confirmatória da tipologia de Schwartz. Estudos de Psicología, 6, 133-142.

Grad, H.M. y Schwartz, S.H. (1998). Aspectos culturales en la estructura de los cuestionarios de valores CVS y RVS. Revista de Psicología Social, 13, 471-483.

Kropp, F., Lavack, A.M. y Holden, S.J.S. (1999). Smokers and beer drinkers: values and consumer susceptibility to interpersonal influence.Journal of Consumer Marketing, 16, $536-557$.

Megías, E., Comas, D., Elzo, J., Megías, I., Navarro, J., Rodríguez, E. y Romaní, O. (2000). Los valores de la sociedad española y su relación con las drogas. Barcelona: Fundación "La Caixa".

Megías, E., Elzo, J., Comas, D., Rodríguez, E., Megías, I., Navarro, J. y Romaní, O. (2001). Valores sociales y drogas. Madrid: FAD.

Menezes, I. y Campos. B.P. (1997). The process of valuemeaning construction: A cross-sectional study. European Journal of Social Psychology, 27, 55-73.
Mercer, G.W. y Kohn, P.M. (1977). Values associated with marijuana use among college students. British Journal of Addiction, 72, 151-158.

Molpeceres, M.A. (1991). Sistemas de valores, estilos de socialización y colectivismo familiar. Un estudio exploratorio de sus relaciones. Tesis de Licenciatura. Citado en J. Pons y E. Berjano, El consumo abusivo de alcohol en la adolescencia: un modelo explicativo desde la psicología social. Madrid: Plan Nacional Sobre Drogas, 1999.

Montero, M. (1994). Indefinición y contradicciones de algunos conceptos básicos en la psicología social. En M. Montero (Comp.), Construcción y Crítica de la Psicología Social (pp. 109-126). Barcelona: Anthropos.

Pons, J. y Berjano, E. (1999). El consumo abusivo de alcohol en la adolescencia: un modelo explicativo desde la psicología social. Madrid: Plan Nacional Sobre Drogas.

Rojo, J., Valdemoro, C. y Cervera, G. (1994). Análisis de los valores en toxicómanos heroinómanos, medidos mediante la escala de valores de Rokeach. Anales de Psiquiatría, 10, 272-278.

Rokeach, M. (1973). The Nature of Human Values. New York: Free Press.

Schwartz, S. (1992). Universals in the content and structure of values: Theoretical advances and empirical tests in 20 countries. Advances in Experimental Social Psychology, 25, 1-65.

Schwartz, S.H. y Bilsky, W. (1987). Towards a universal psychological structure of human values. Journal of Personality and Social Psychology, 5, 550-562.

Schwartz, S.H. y Bilsky, W. (1990). Towards a theory of the universal content and structure of values: Extensions and cross-cultural replications. Journal of Personality and Social Psychology, 58, 878-891.

Toler, C. (1975). The personal values of alcoholics and addicts. Journal of Clinical Psychology, 31, 554-557.

Unger, J.B., Ritt-Olson, A., Teran, L., Huang, T., Hoffman, B.R. y Palmer, P. (2002). Cultural values and substance use in a multiethnic sample of California adolescents. Addiction Research \& Theory, 10, 257-279. 